\title{
Identification of differentially expressed IncRNAs involved in transient regeneration of the neonatal C57BL/6J mouse heart by next-generation high-throughput RNA sequencing
}

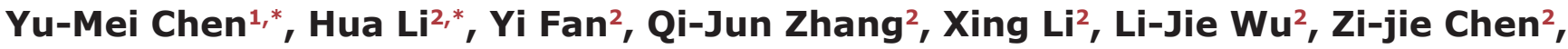 \\ Chun Zhu ${ }^{3}$, Ling-Mei Qian ${ }^{2}$ \\ ${ }^{1}$ Department of Emergency, Zhongshan Hospital, Fudan University, Shanghai 200032, P.R. China \\ ${ }^{2}$ Department of Cardiology, The First Affiliated Hospital of Nanjing Medical University, Nanjing 210029, P. R. China \\ ${ }^{3}$ Department of Pediatrics, Obstetrics and Gynecology Hospital Affiliated to Nanjing Medical University, Nanjing, Jiangsu \\ 210004, P. R. China \\ *These authors contributed equally to this work
}

Correspondence to: Ling-Mei Qian, email: Imqian@njmu.edu.cn Chun Zhu, email: zhifangxibao@163.com

Keywords: neonatal mouse, heart, regeneration, IncRNAs

Received: August 15, 2016

Accepted: February 20, 2017

Published: March 03, 2017

Copyright: Chen et al. This is an open-access article distributed under the terms of the Creative Commons Attribution License (CC-BY), which permits unrestricted use, distribution, and reproduction in any medium, provided the original author and source are credited.

\section{ABSTRACT}

Previous studies have shown that mammalian cardiac tissue has a regenerative capacity. Remarkably, neonatal mice can regenerate their cardiac tissue for up to 6 days after birth, but this capacity is lost by day 7. In this study, we aimed to explore the expression pattern of long noncoding RNA (IncRNA) during this period and examine the mechanisms underlying this process. We found that 685 IncRNAs and 1833 mRNAs were differentially expressed at $\mathrm{P} 1$ and $\mathrm{P7}$ by the next-generation high-throughput RNA sequencing. The coding genes associated with differentially expressed IncRNAs were mainly involved in metabolic processes and cell proliferation, and also were potentially associated with several key regeneration signalling pathways, including PI3K-Akt, MAPK, Hippo and Wnt. In addition, we identified some correlated targets of highly-dysregulated IncRNAs such as Igfbp3, Trnp1, Itgb6, and Pim3 by the codingnoncoding gene co-expression network. These data may offer a reference resource for further investigation about the mechanisms by which IncRNAs regulate cardiac regeneration.

\section{INTRODUCTION}

In recent years, the traditional view that the heart is a terminally differentiated organ with no capacity for cardiomyocyte renewal has been emphatically refuted by an increasing number of studies in humans and other mammals [1-3]. Several studies have found that 1-dayold neonatal mice were capable of undergoing cardiac regeneration following multiple types of cardiac injury, but the ability to efficiently regenerate heart muscle was lost by postnatal day 7 [4-6]. Further study by our group examined the global gene expression patterns of the neonatal mouse heart at key time points ( 1 and 7 days old) [7] and found several differentially expressed genes involved in transient regeneration of the neonatal mouse heart. However, there is limited original data regarding cardiac regeneration during this developmental period.

Long noncoding RNAs (lncRNAs) are defined as transcripts greater than $200 \mathrm{nt}$ without a known proteincoding function [8]. There is increasing evidence that IncRNAs can regulate gene expression at the epigenetic, transcription, and post-transcription levels and participate in a range of biological processes [9]. A recent study showed that many lncRNAs are expressed in the heart and confirmed the essential role of lncRNAs in the cardiovascular system such as modulating cardiac development [10]. Further studies have shown that IncRNAs are involved in coronary artery disease [11] and myocardial hypertrophy [12]. In addition, some IncRNAs have been found to be involved in cardiac 
regeneration. For example, lncRNA-ST8SIA3 (also named lncRNA ROR, regulator of reprogramming) enhances the reprogramming of fibroblasts to become induced pluripotent stem cells or cardiomyocytes [13]. Knocking out the gene lncRNA-H19 has been shown to promote differentiation of parthenogenetic embryonic stem cells to cardiomyocytes with strong heart-like beating [14]. However, our understanding of the role of lncRNAs in regeneration of heart tissue in mice is still limited. Therefore, two key time points ( 1 and 7 days old) were selected for the analysis of global lncRNA expression profiles in C57BL/6 mice using next-generation highthroughput RNA sequencing techniques.

\section{RESULTS}

\section{Profiles of the differently expressed IncRNAs and mRNAs}

From the IncRNA expression profiles, 685 differentially expressed lncRNAs in mouse cardiac tissue were compared between P1 and P7. Compared to 1-dayold cardiac tissue, we identified 418 downregulated lncRNAs and 267 upregulated lncRNAs in 7-day-old cardiac tissue. As shown in Figure 1A, these lncRNAs showed a general difference between P1 and P7. 32 lncRNAs exhibited a high fold change of at least 3-fold, where 9 lncRNAs exhibited increased expression and 23 lncRNAs decreased expression (Supplementary Material). The the 685 differentially expressed lncRNAs in mouse cardiac tissue were list in the supplementary materials.

From the analysis of sequencing data, 1833 differentially expressed mRNAs were found with comparisons between 1-day-old and 7-day-old cardiac tissue, among which 859 were upregulated mRNAs and 974 were downregulated mRNAs in 7-day-old cardiac tissue compared with tissue from 1-day-old mice. Their distinct expression patterns are also presented in the hierarchical clustering analysis shown in Figure 1B.

\section{Genomic location of differentially expressed IncRNAs}

Length of the IncRNAs was generally between 400 bp and 3600 bp (Figure 2A, 2B). Chromosomal distribution showed the numbers of up- and downregulated lncRNAs located on different chromosomes (Figure 2C, 2D). Chromosomal distribution showed the upregulated lncRNAs were mainly located on chromosome 14, 1 and 6 , and the downregulated lncRNAs were mainly located on chromosome 17, 9,6 and 7.The functional characterisation of these lncRNAs presents a formidable task, and some studies suggest that lncRNAs regulate higherorder chromosomal dynamics, sub-cellular structural organisation and telomere biology [19]. LncRNAs have been suggested to originate from complex loci that include
lncRNA and associated protein-coding genes, since several lncRNAs have been reported to regulate the expression of adjacent protein-coding genes [20,21]. Such information about these lncRNAs and their nearby coding genes might be useful to predict their functional roles in heart regeneration (Table 1 ).

\section{Validation of differentially expressed IncRNAs}

To verify the RNA-Seq data, we randomly selected 22 differentially expressed lncRNAs for qPCR analysis, among which 11 were upregulated lncRNAs and 11 were downregulated lncRNAs in 7-day-old cardiac tissue compared with tissue from 1-day-old mice. The result showed that the expression patterns of those lncRNAs were consistent with the RNA-Seq data (Figure 3).

\section{GO and pathway analysis}

We performed GO analysis for the coding genes associated with differentially expressed lncRNAs as shown in Figure 4A. The top 10 terms associated with biological processes were: (1) GO:0008283: cell proliferation; (2) GO:0030154: cell differentiation; (3) GO:0042127: regulation of cell proliferation; (4) GO:0044249: cellular biosynthetic process; (5) GO:0019222: regulation of metabolism; (6) GO:0042981: regulation of apoptosis; (7) GO:0016049: cell growth; (8) GO:0060038: cardiac muscle cell proliferation; (9) GO:0007346: regulation of the mitotic cell cycle; and (10) GO:0051726: regulation of the cell cycle.

We also performed KEGG pathway enrichment analysis for the coding genes associated with differentially expressed lncRNAs. The most significant pathways were: metabolic, tight junctions and signalling pathways including PI3K-Akt, cGMP-PKG, cAMP, MAPK, calcium, adrenergic signalling in cardiomyocytes, Hippo and Wnt (Figure 4B).

\section{CNC network}

Biological networks cover modules of genes or proteins that may function in the same pathway; genes or proteins inside the same module can be co-regulated [22]. Therefore, construction of a CNC network could infer the biological functions of lncRNAs [22, 23], which would increase our understanding of the complex molecular mechanisms of heart regeneration.

Our data showed that the downregulated coexpression network was composed of 74 network nodes and 328 connections between 18 lncRNAs and 67 coding genes. The upregulated co-expression network was composed of 51 network nodes and 158 connections between 17 lncRNAs and 43 coding genes. In both of the co-expression networks, there was a positive correlation between most of the pairs. In addition, we observed that 
one lncRNA could target several coding genes and that one coding gene correlated with several lncRNAs. For example, in the downregulated co-expression network, the downregulated IncRNAs fantom3_B230352I09 and fantom3_1110003P13 were correlated with 17 and 15 coding genes respectively. In the upregulated coexpression network, lncRNA fantom3_2210409C20 and fantom3_G730023M12, upregulated in P7, were coexpressed with 8 and 7 coding genes respectively. By the coding-noncoding gene co-expression network, we identified some correlated targets of highly-dysregulated lncRNAs such as Igfbp3, Trnp1 Itgb6, and Pim3. As shown in Figure 5, those lncRNAs with the highest coexpression show the most important position.

\section{DISCUSSION}

Although various miRNAs [24], transcription factors [25], growth factors [26], and cell cycle regulatory elements $[27,28]$ have been shown to regulate the genes that orchestrate proliferation during the regeneration of cardiomyocytes, the role of lncRNAs is poorly understood. Compared with adult mammals, neonatal mice have full capacity for cardiac regeneration after injury. Therefore, charting the transcriptional profile of lncRNAs in regenerative hearts from neonatal mice is a key step in understanding the role of lncRNAs, and this study was the first to examine the expression of lncRNAs in regenerative hearts.

Using next-generation high-throughput RNA sequencing techniques, this study found 685 lncRNAs that were differentially expressed in the heart between postnatal days 1 and 7 (P1 and P7, respectively), which consisted of 418 downregulated and 267 upregulated lncRNAs. In addition, 1833 differentially expressed mRNAs were identified, consisting of 859 upregulated and 974 downregulated mRNAs. 11 upregulated lncRNAs and 11 downregulated $\operatorname{lncRNAs}$ were further validated using qRT-PCR, and the results were consistent with the data of RNAseq. In addition, chromosomal distribution showed the upregulated lncRNAs were mainly located on chromosome 14, 1 and 6, and the downregulated lncRNAs were mainly located on chromosome 17, 9, 6 and 7 . Interestingly, the Notch1 located on the chromosome 9 and TBX2 located on the chromosome 17 are closely related to heart development $[29,30]$.

We also used GO and pathway analyses to identify potential biological functions enriched among the associated genes of differentially expressed lncRNAs. GO functional enrichment analysis showed that the coding

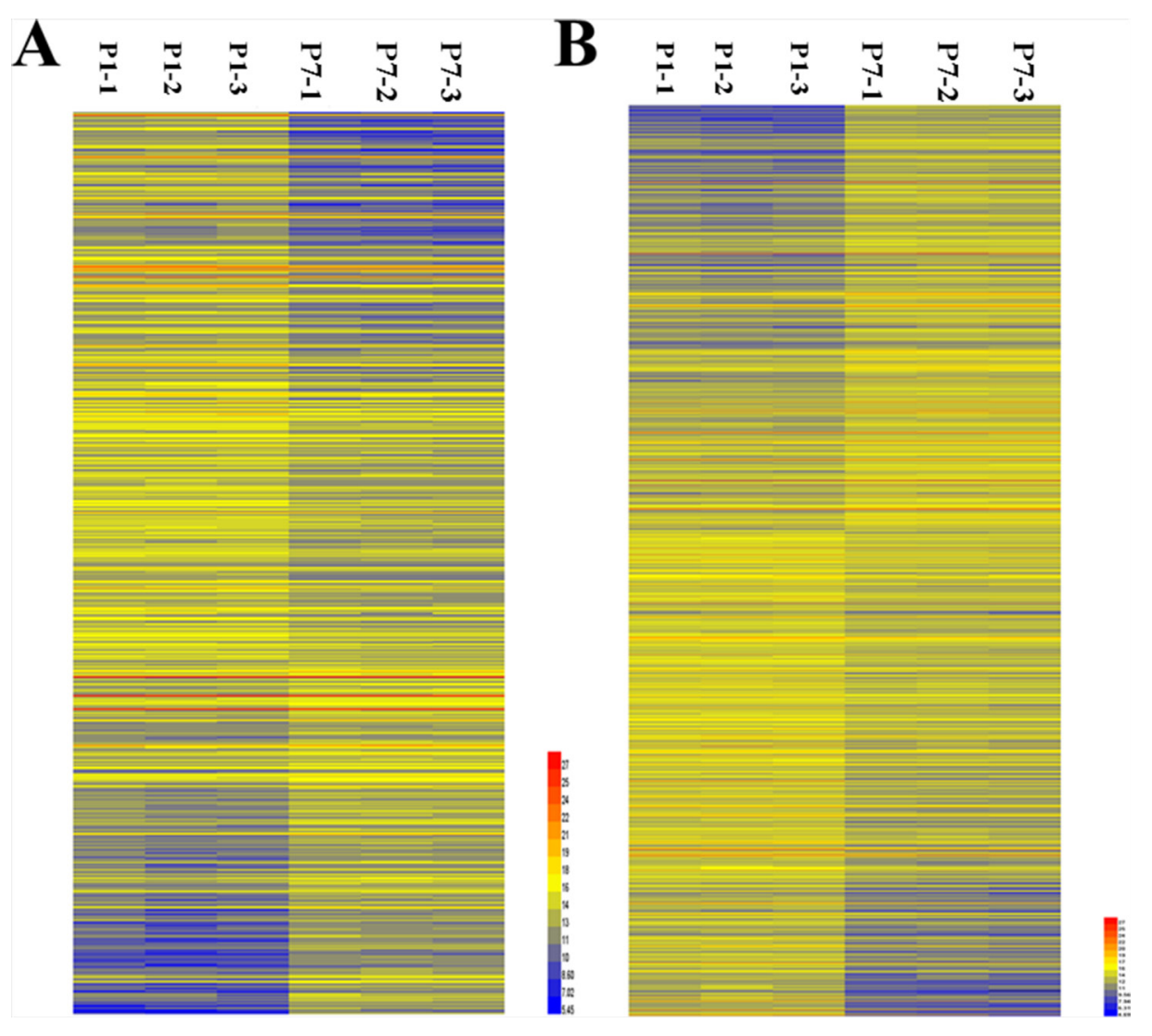

Figure 1: Hierarchical clustering of the differentially expressed LncRNAs and mRNAs in murine cardiac tissue in postnatal days 1 and 7 (P1 and P7). (A) Differentially expressed LncRNAs; (B) Differentially expressed mRNAs. Yellow indicates upregulation; blue indicates downregulation. 
genes associated with differentially expressed lncRNAs were significantly involved in the following processes: cell proliferation and its regulation, cell differentiation, the cellular biosynthetic process; regulation of metabolism, regulation of apoptosis, cell growth, cardiac muscle cell proliferation and regulation of the mitotic and cell cycles. Stimulating endogenous cardiac regeneration by increasing the cell cycle activity of cardiomyocytes could increase the number of cardiomyocytes in an injured heart [31]. Previous studies have suggested that lncRNAs play a role in cell cycle regulation and cardiac development [32-34] and therefore that they are involved in cell cycle regulation of cardiomyocytes and cardiac regeneration [35]. Our study provides further evidence of the potential role of differentially expressed lncRNAs in the regenerative potential of heart tissue in neonatal mice, via regulation of the differentially expressed genes associated with differentiation and proliferation of cardiomyocytes.

Pathway-Express analysis identified that the metabolic pathways were most significantly affected by the coding genes associated with differentially expressed lncRNAs in 7-day-old cardiac tissue compared with tissue from 1-day-old mice. Macromolecules including glycogen, proteins, nucleotides and lipids represent both the cellular energy reserve and also the essential building blocks for cell renewal [36]. Neonatal mice retain the capacity for cardiac regeneration, but this is lost after 7 days. Therefore, the demand for the anabolic production of macromolecules in the regenerating heart increases during the first 6 days after birth.

The other significant signalling pathways were thePI3K-Akt, MAPK, Hippo, and adrenergic signalling in cardiomyocytes. Previous studies have shown that MAPK signalling pathways have non-redundant roles in the regulation of zebrafish cardiac regeneration [37]. The PI3K-AKT pathway promotes cardiomyocyte proliferation, survival, and physiological hypertrophy [38], and both the Hippo and Wnt signalling pathways also play an important role in cardiac regeneration [38-40]. In addition, it has been reported that sympathetic nerves have an important role in neonatal mammalian heart regeneration $[41,42]$. Therefore, we hypothesised that those differentially expressed lncRNAs might participate in the process of neonatal cardiac regeneration by regulating the associated gene expression targeting these pathways.

To study the biological functions and potential mechanisms of differentially expressed lncRNAs, we

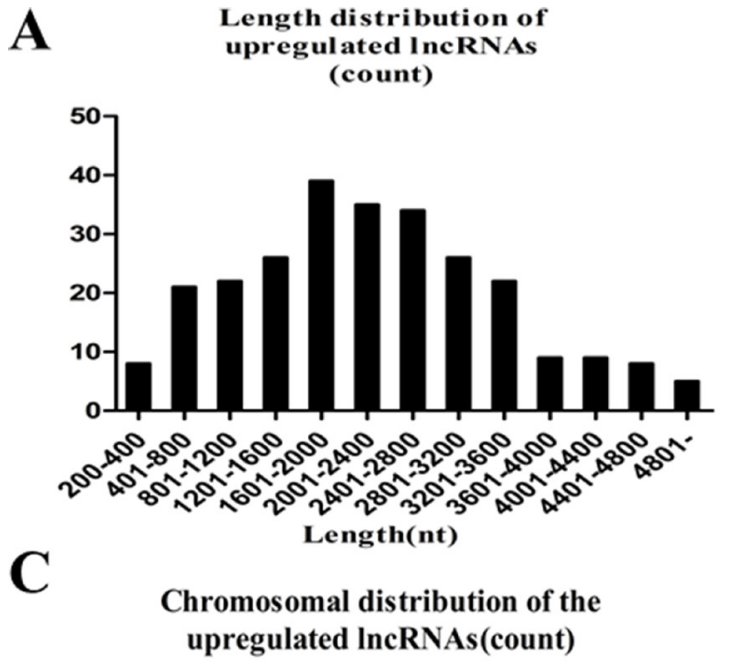

B
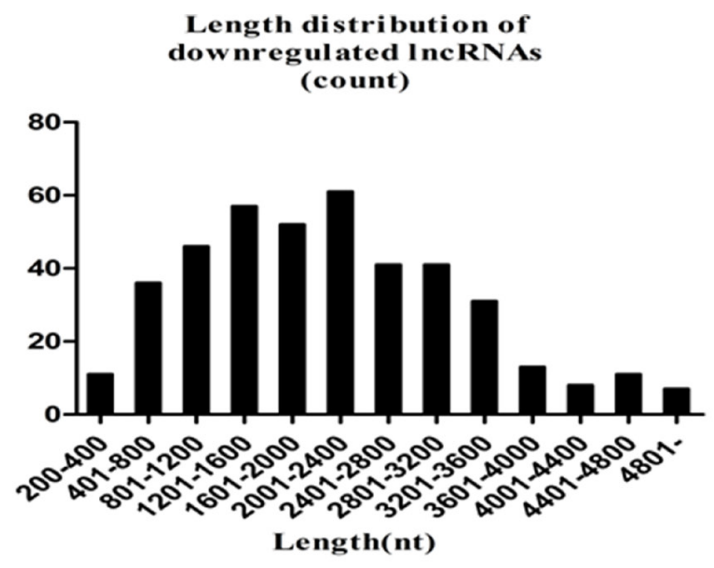

D
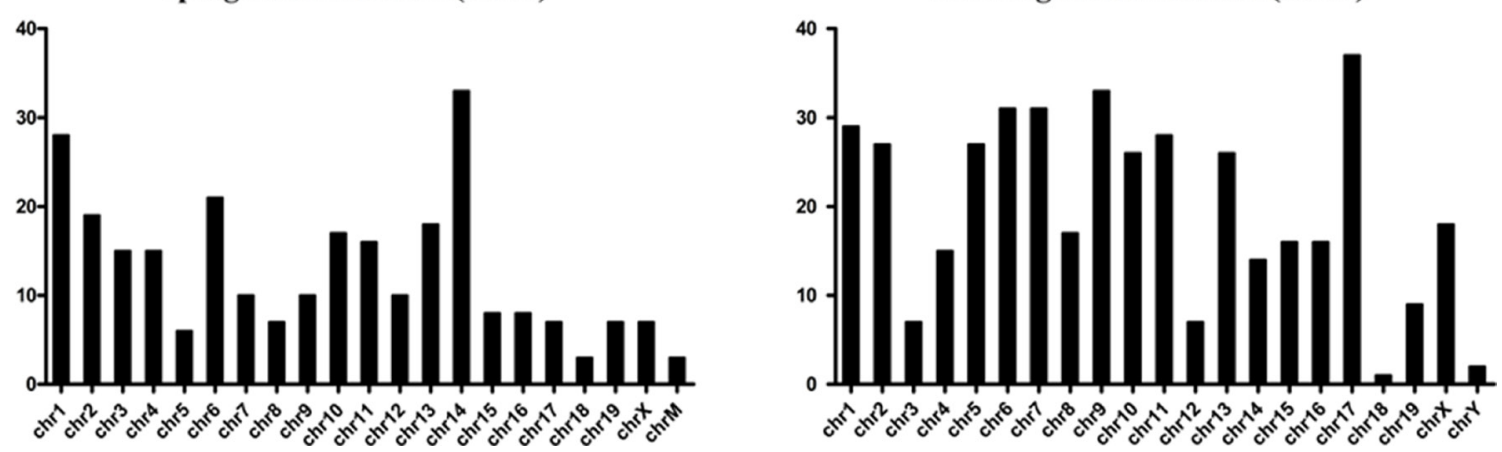

Figure 2: (A) Length distribution of the upregulated lncRNAs. (B) Length distribution of the downregulated lncRNAs. The upregulated and downregulated lncRNAs are mainly between 400 and 3600 bp in length. (C) Chromosome distribution shows the numbers of upregulated lncRNAs located in different chromosomes; chr represents chromosome. (D) The numbers of downregulated lncRNAs located in different chromosomes. 
Table 1: IncRNAs and their nearby coding genes

\begin{tabular}{|c|c|c|c|c|}
\hline Transcript & Upstream second gene & Upstream first gene & Downstream first gene & Downstream second gene \\
\hline fantom3_B430306C15 & fantom3_9230114P20 & Slc10a6 & 1700016H13Rik & fantom3_F830205C23 \\
\hline NR_033497 & fantom3_4930408C10 & fantom3_A830009F23 & Myh7 & fantom3_D330040O14 \\
\hline fantom3_G630025L12 & Kctd6 & Acox 2 & fantom3_C630031G01 & Fam107a \\
\hline fantom3_D330013N04 & fantom3_A330004D21 & Myl4 & Itgb3 & fantom3_7030418D15 \\
\hline fantom3_B230377K03 & Mt3 & fantom3_A830083K16 & Mt2 & fantom3_1810074C23 \\
\hline fantom3_1110003P13 & 2700060E02Rik & fantom3_8030491K24 & Gng2 & fantom3_C130093P08 \\
\hline fantom3_4930408C10 & Myh6 & Mir208a & fantom3_A830009F23 & D830015G02Rik \\
\hline fantom3_9530030H05 & Oxnad1 & fantom3_2410002F01 & Msmb & Ncoa4 \\
\hline NR_033813 & fantom3_A330045P15 & Begain & Meg3 & fantom3_I0C0047O18 \\
\hline fantom3_2310031C01 & Fam $47 \mathrm{e}$ & fantom3_D530019K15 & Stbd 1 & Ccdc158 \\
\hline fantom3_D530019K15 & fantom3_C730043O17 & Fam $47 \mathrm{e}$ & fantom3_2310031C01 & Stbd1 \\
\hline fantom3_2510038C20 & Mt2 & fantom3_1810074C23 & Mt1 & Nup93 \\
\hline fantom3_1810074C23 & fantom3_B230377K03 & Mt2 & fantom3_2510038C20 & Mt1 \\
\hline NR_030416 & fantom3_I0C0030C13 & fantom3_1100001A04 & fantom3_D130045N19 & Igf2 \\
\hline NR_001592 & fantom3_9530074L01 & fantom3_9830166K08 & fantom3_I0C0030C13 & fantom3_1100001A04 \\
\hline fantom3_I0C0030C13 & fantom3_9830166K08 & H19 & fantom3_1100001A04 & Mir675 \\
\hline fantom3_1100001A04 & H19 & fantom3_I0C0030C13 & Mir675 & fantom3_D130045N19 \\
\hline fantom3_9830143G06 & Tmem164 & fantom3_6030487A22 & fantom3_4932428A02 & fantom3_B230314K16 \\
\hline fantom3_4932428A02 & fantom3_6030487A22 & fantom3_9830143G06 & fantom3_B230314K16 & fantom3_C430013O12 \\
\hline fantom3_9230118C23 & fantom3_D330009G10 & 2310050B05Rik & fantom3_2310050B05 & Nmrk2 \\
\hline NR_015477 & Dapk3 & fantom3_D330009G10 & fantom3_9230118C23 & fantom3_2310050B05 \\
\hline fantom3_D330009G10 & Snord37 & Dapk3 & 2310050B05Rik & fantom3_9230118C23 \\
\hline fantom3_D130045N19 & fantom3_1100001A04 & Mir675 & Igf2 & Mir483 \\
\hline fantom3_1010001E19 & fantom3_B230374I20 & fantom3_B230350D20 & fantom3_E430001N13 & fantom3_D830012D22 \\
\hline fantom3_D830012D22 & fantom3_1010001E19 & fantom3_E430001N13 & fantom3_0610011H19 & fantom3_D030018G08 \\
\hline fantom3_E430001N13 & fantom3_B230350D20 & fantom3_1010001E19 & fantom3_D830012D22 & fantom3_0610011H19 \\
\hline fantom3_G730023M12 & fantom3_A730057F09 & Stc2 & fantom3_F830230J18 & Bod1 \\
\hline fantom3_4930578005 & fantom3_4930558J22 & Hist1h4b & Hist1h4a & Hist1h3a \\
\hline fantom3_A830073O21 & Gm4971 & fantom3_A730020G15 & Fam174b & fantom3_4932443D20 \\
\hline fantom3_2310024F14 & fantom3_A730026F12 & Fxyd6 & Fxyd2 & fantom3_1700101G12 \\
\hline fantom3_A630066012 & Ank1 & fantom3_6330529I17 & Mir486 & Mir3107 \\
\hline NR_039592 & fantom3_E030018F15 & $\operatorname{Kctd} 12$ & fantom3_4933432I03 & 4933432I03Rik \\
\hline fantom3_D630044L13 & fantom3_F930015M13 & Mir3966 & fantom3_9530076L14 & Den \\
\hline fantom3_1700119I11 & Zcchc9 & Ckmt2 & fantom3_6430599K20 & fantom3_D830014B08 \\
\hline fantom3_C130052N24 & Stard4 & fantom3_E230006P11 & Nrep & fantom3_D930015L03 \\
\hline fantom3_D830028G10 & Lrtm1 & fantom3_A930039P05 & fantom3_D130049P09 & fantom3_B930020018 \\
\hline fantom3_A230064C13 & Crhrl & Sppl2c & Mapt & Kansl1 \\
\hline fantom3_2210409C20 & Itgb6 & fantom3_E030019H01 & fantom3_F420008A03 & fantom3_I920190L12 \\
\hline fantom3_2310015A16 & Sik1 & fantom3_C130051M16 & fantom3_C130017E03 & Hsf $2 b p$ \\
\hline fantom3_B230352I09 & fantom3_C230086B22 & fantom3_4933422O15 & fantom3_2010321I05 & Tnrc6a \\
\hline
\end{tabular}

constructed a CNC network on the basis of the correlation analysis between the differentially expressed lncRNAs and mRNA. Compared with the conventional differential expression analysis, the co-expression network analysis indicates the higher-order relationships of the transcripts in the biological framework [43]. In our co-expression network, we observed that some lncRNAs expression levels were markedly correlated with the expression of several protein-coding genes, indicating the importance of their location. For instance, the lncRNA fantom3 B230352I09, which was downregulated in P7, was coexpressed with 17 coding genes, most of which were 


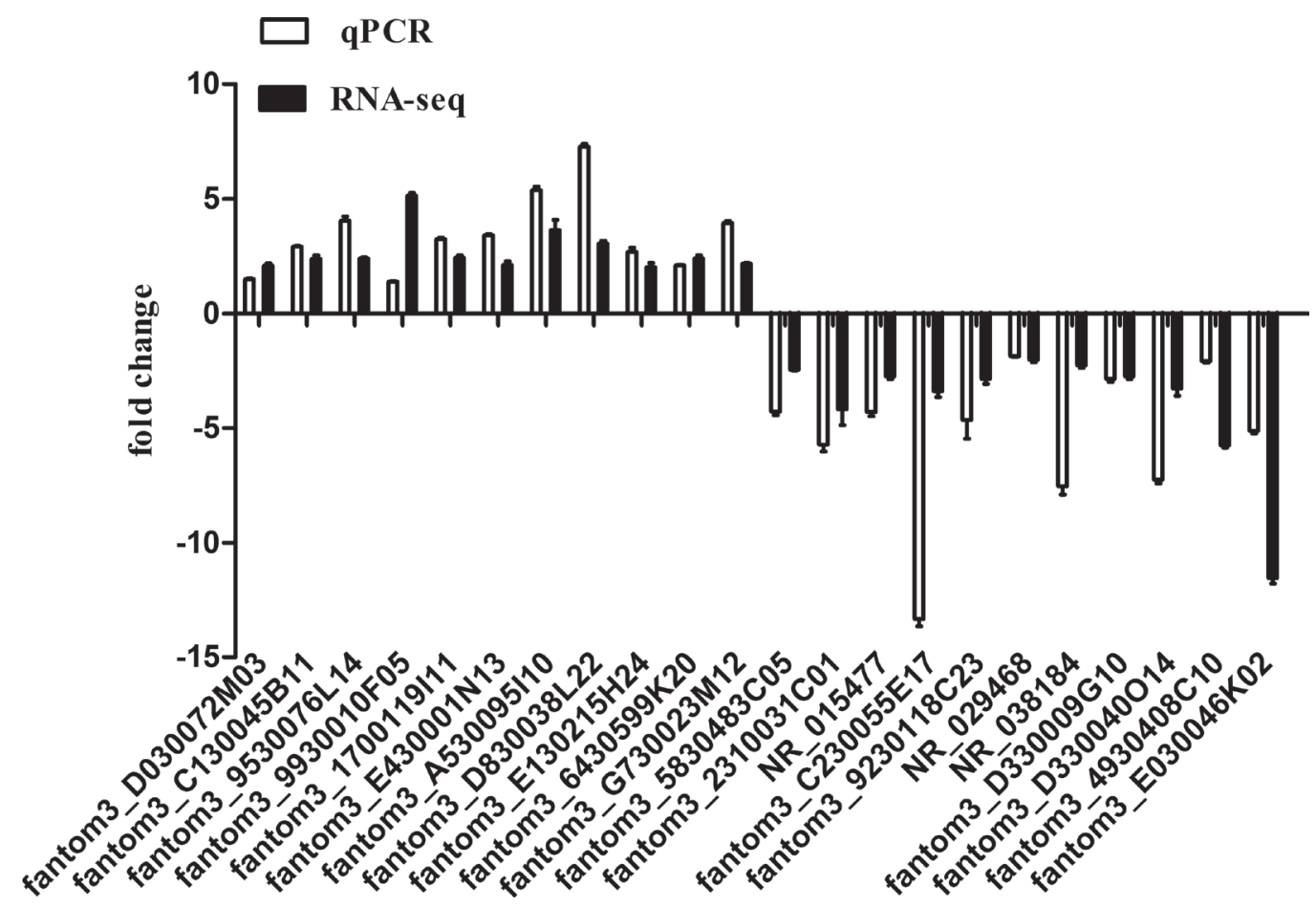

Figure 3: Comparison between sequencing data and the qRT-PCR result for IncRNAs. Columns above the X-axis indicate the upregulated lncRNAs, and columns below the X-axis represent the downregulated lncRNAs.-PCR. The validation results indicated that the sequencing data correlated well with the qPCR results.

A

sig GO terms of DE gene-BP

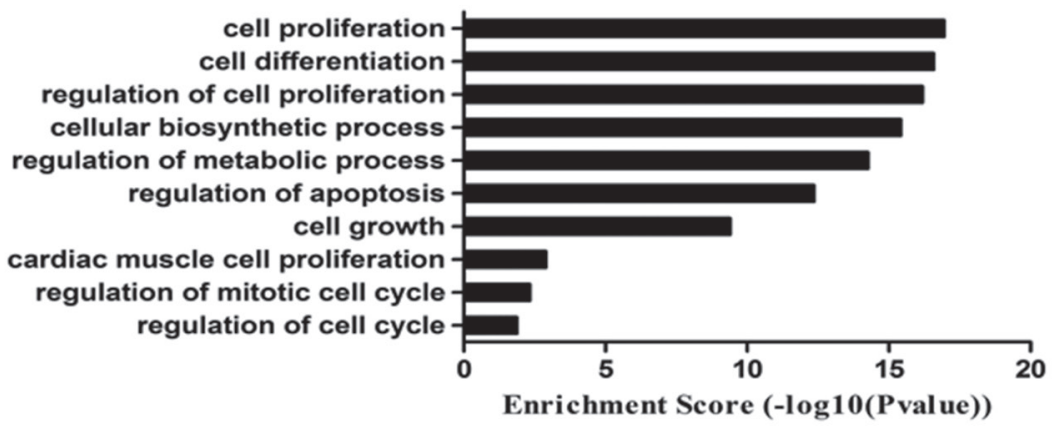

B

sig pathway of DE gene

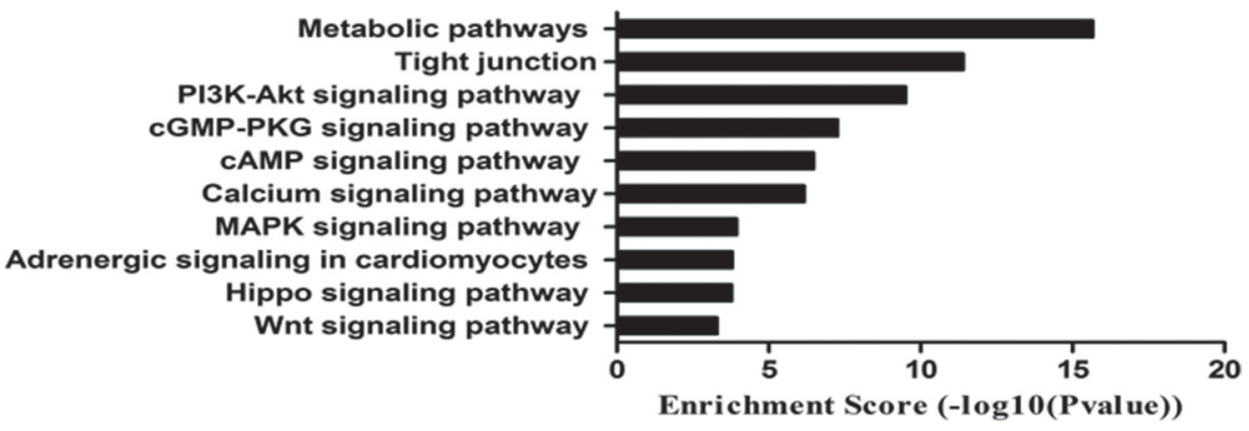

Figure 4: GO and pathway analysis. (A) The list of the top $10 \mathrm{GO}$ terms with the associated coding gene function of dysregulated IncRNAs. (B) The list of the top 10 pathways associated with the coding gene of dysregulated lncRNAs. 
related to apoptosis, cell growth, regulation of the cell cycle and cell proliferation. As another example, the Trnp1 gene [44] has been reported to accelerate cell cycle progression and the Pim3 gene[45] has a cardio-protective effect through the process of antiapoptosis. Igfbp3 is a downstream target of Wnt signaling pathway and participates in the regulation of adult cardiac progenitor cell regeneration [46].The lncRNA fantom3_2210409C20, upregulated in P7, was co-expressed with 8 coding genes. Among the coexpressed genes, Itgb6 (a $\beta$ subunit of integrins) was found to be relatively close to fantom3_2210409C20. Integrins are expressed in all cellular compartments of the cardiovascular system and mediate cell adhesion, migration, proliferation and survival in many cell types [47]. Whether cardiac regeneration in 1-day-old neonatal mice is regulated by these lncRNAs warrants further study. Although our current understanding of lncRNA regulation in cardiac regeneration is in its infancy, several approaches can be employed to investigate their biological functions, including over-expression of lncRNA, lncRNA silencing and structure disruption, which may provide more conclusive evidence to explain the regulatory mechanisms.

In conclusion, we have shown the global different expression profiles of lncRNAs during the first seven days in the neonatal mouse heart. By bioinformatics prediction, we obtained some target genes of the candidate lncRNAs such as Igfbp3, Trnp1 Itgb6, and Pim3, which correlated with the process of proliferation. Collectively, our results suggest that lncRNAs could be important regulators in mammalian cardiac regeneration. Further work is needed to understand the biological functions and molecular mechanisms of specific lncRNAs implicated in cardiac regeneration.

\section{MATERIALS AND METHODS}

\section{Animal and tissue preparation}

The neonatal C57BL/6J mice were purchased from the Model Animal Research Centre of Nanjing University (Nanjing, China).Our study was approved by the Animal Care and Use Committee of Nanjing Medical University (Nanjing, China) and the methods were carried out in accordance with the approved guidelines. The left ventricular apex was removed from neonates 1 and 7 days after birth, cleaned and snap-frozen in liquid nitrogen. Three biological replicates were generated per timepoint. Hearts from five to ten mouse pups were pooled for each biological replicate.

\section{RNA extraction and quality control}

Total RNA was extracted from neonatal mouse heart tissue using TRIzol reagent (Invitrogen Life Technologies) in accordance with the manufacturer's protocol. RNA quantity and quality were measured using a NanoDrop ND-1000. RNA integrity was assessed by standard denaturing agarose gel electrophoresis.

\section{Analysis of sequencing data}

RNA-Seq based transcriptome profiling was performed by the Guangzhou RiboBio Co, using the high-throughput, high-sensitivity HiSeq 3000 sequencing platform (Illumina Company, USA). Clean data were acquired by removing joint sequence fragments, lowquality segments and ribosomal RNA. The short reads were mapped to the reference genome using TopHat [15] with 2 mismatches (--read-mismatches $=2)$ and 2 gaps
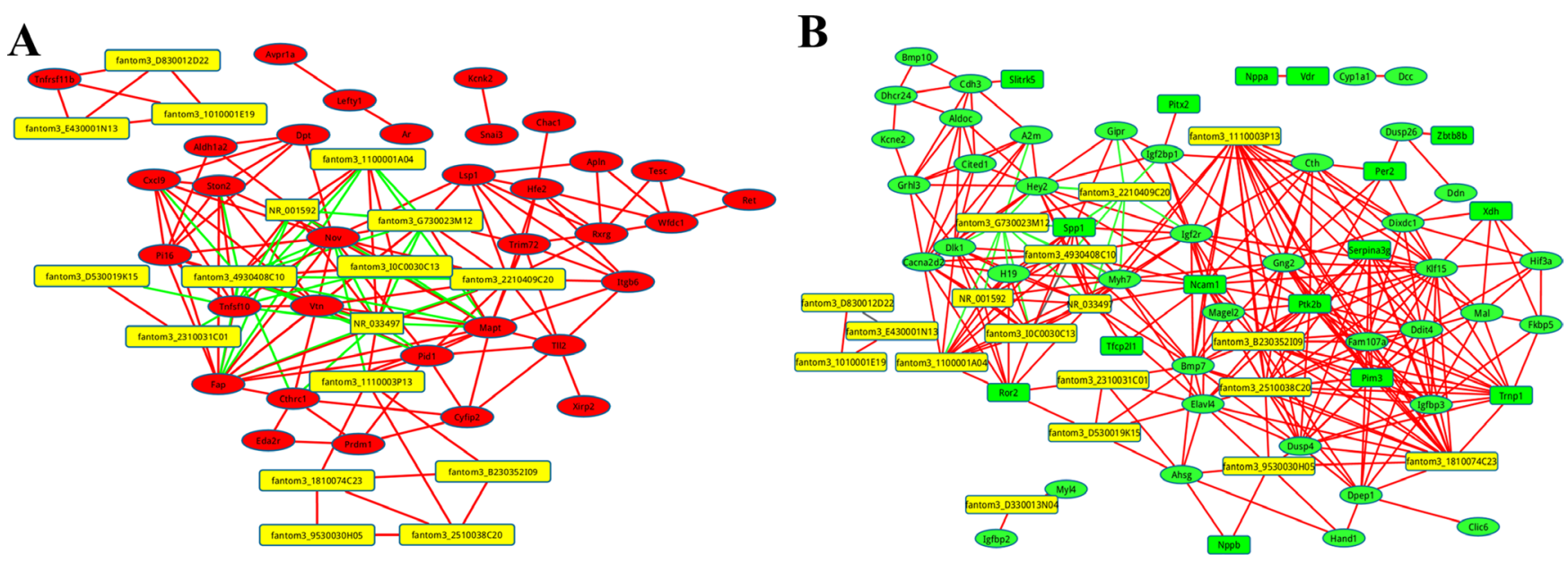

Figure 5: The co-expression network of the coding genes comparing P1 and P7, with the highly-dysregulated IncRNAs. A correlation of $>0.99$, or a correlation $<-0.99$ and $P$ value of $<0.05$ were recognised as co-expression. Red lines indicate a positive correlation, green lines indicate a negative correlation. (A) The co-expression network of the upregulated coding genes. A red circle indicates the upregulated coding genes, and a yellow square indicates lncRNAs. (B) The co-expression network of the downregulated coding genes. A green circle indicates the downregulated coding genes, a yellow square indicates LncRNAs. 
Table 2: PCR primer sequences used

\begin{tabular}{|c|c|c|}
\hline Gene & Forward primer $\left(5^{\prime}-3^{\prime}\right)$ & Reverse primer $\left(3^{\prime}-5^{\prime}\right)$ \\
\hline fantom3_E030046K02 & CTGGAAGAGAGCACCAGACC & TGGTTTCTCACACCAGACCA \\
\hline fantom3_4930408C10 & TGGGGAGGAGAGAAAAGGTT & TGTGCCTCTCCCTTTCTGTT \\
\hline fantom3_2310031C01 & ACTCCCCAAGTGTGCTTTTG & TGTACAGTTCAAGGCTCTGTTTT \\
\hline NR_015477 & AGCGAGGCCAGCAGTCTAC & GGCCTCAGAATCCATGCTT \\
\hline fantom3_C230055E17 & AAGCCTCTATGGCAAGCGTA & AAGGCTGACCAAAAGCAGAA \\
\hline fantom3_9230118C23 & AGGTCCCAAGAGCACAGAGA & CAGGTATGAGGCCCTTGTGT \\
\hline fantom3_5830483C05 & TGCTGGTCACAAAAGTCAGG & GGTCGGTGGAGGAGTATGAA \\
\hline NR_029468 & GATTTTTCCGTCTGGTCCAA & CAGGCCGTCTTCTCAGACTC \\
\hline NR_038184 & CTATCCACCACACCCTGCTT & ССТTCTACATTCCCCACGAA \\
\hline fantom3_D330009G10 & AGCGAGGCCAGCAGTCTAC & GGCCTCAGAATCCATGCTT \\
\hline fantom3_D330040O14 & TAGCCAGAGTTGGTGTGCAG & AATGGCTTTGGTGTCTTTGG \\
\hline fantom3_9930010F05 & TATGCAATACCATGCCCAGA & CTTGGCTCCACTTCAAGAGC \\
\hline fantom3_1700119I11 & AGCATGGCTAACGGACAGAT & CCCATGATGCAAGATTTCCT \\
\hline fantom3_E430001N13 & GTACCGCAAGGGAAAGATGA & CCAGCTATCACCAAGCTCGT \\
\hline fantom3_A530095I10 & CCCTGCCCTGTAATTTTGAA & TATCCGGCTCTGCAAGAAGT \\
\hline fantom3_D830038L22 & GCTTTGCGTTTTCTGAGTCC & CATCCCTTTGCACTGTCCTT \\
\hline fantom3_6430599K20 & TTCCTTGGCTCCTAGCAGAA & CTATGCCCTTGTCTGGCAAT \\
\hline fantom3_9530076L14 & TGCCTACTTCTGCAACACCA & CССАСССТСАССТСАТАGTG \\
\hline fantom3_G730023M12 & CAAGTGCTGAAGGATGACCA & GCTGGAGAGGCTCAATCATC \\
\hline fantom3_D030072M03 & AACCAAGGCCAAGATTCCTT & GAGCCAGGCAATGAACAAAT \\
\hline fantom3_E130215H24 & AGGCAGCGCTCTGAGATAAG & CTGCGGGTGATTTATGTGTG \\
\hline fantom3_C130045B11 & GGCCTTCTGTTTGTGTGGTT & GCTTTCCACTCTCTGGCATC \\
\hline GAPDH & ATTCAACGGCACAGTCAA & CTCGCTCCTGGAAGATGG \\
\hline
\end{tabular}

(read-gap-length $=2$ ). Transcripts were assembled and the relative abundance of genes was estimated using Cufflinks in reads per kilobase of transcript per million mapped reads (RPKM) [16]. Differentially expressed lncRNAs between P1 and P7 were identified through fold change as well as $P$ values calculated with a $t$-test. The threshold set for dysregulated lncRNAs was a fold change of $\geq 1.0$ and a $P$ value of $\leq 0.05$. A hierarchical clustering analysis was applied to classify lncRNAs based on their expression levels.

\section{Quantitative PCR (qPCR) analysis}

The results of RNA-Seq were validated via qPCR analysis. The cDNA was generated from $1 \mu \mathrm{g}$ of total RNA using an AMV Reverse Transcriptase kit (Invitrogen Life Technologies). We designed primers for each lncRNA using Primer 3 (http://sourceforge.net/projects/primer3/) and used the Basic Local Alignment Search Tool from NCBI to verify that the amplified product was unique. The qPCR was performed using an ABI 7300 Sequence Detection system (Applied Biosystems, Foster City, CA, USA), under the following conditions: denaturation at $95^{\circ} \mathrm{C}$ for $10 \mathrm{~min}$, and 40 cycles of $95^{\circ} \mathrm{C}$ for $15 \mathrm{sec}$ and $60^{\circ} \mathrm{C}$ for $1 \mathrm{~min}$. Relative gene expression levels were normalised to GAPDH and calculated according to the $2^{-\Delta \mathrm{CT}}$ method. Each sample was measured in triplicate and reproduced at least three times. The sequences of the primers used are shown in Table 2.

\section{GO and pathway analysis}

Gene ontology (GO) analysis provides a controlled vocabulary to describe the molecular actions of gene products, their cellular locations and the biological processes in which those actions occur [17]. Fisher's exact test was used to detect any significant overlap between the differentially expressed list and the GO annotation list. The $P$ value denotes the significance of GO term enrichment between differentially expressed genes (a $P$ value $\leq 0.05$ is accepted as significant). Pathway analysis was used to map genes to KEGG pathways. The $P$ value (calculated via EASE-score, Fisher $P$ value or hypergeometric $P$ value) denotes the significance of the pathway correlations (a $P$ value of $\leq 0.05$ is recommended, since the smaller the $P$ value, the greater the significance of the pathway). 


\section{Construction of the coding-noncoding gene co-expression network}

The coding-noncoding gene co-expression network (CNC network) was constructed based on the correlation analysis between the differentially expressed lncRNAs and mRNAs. The algorithm has been previously described [18]. Briefly, for each pair of genes analysed, we calculated the Pearson correlation coefficient (PCC) and correlation coefficient of the PCC between lncRNAs and coding genes (lncRNA-mRNA PCC and lncRNAlncRNA PCC). We subsequently selected lncRNAs and mRNAs based on the PCC using the selection parameter PCC $\geq 0.99$ as significant and created the co-expression networks using Cytoscape (Institute of Systems Biology, Seattle, US).

\section{Statistical analysis}

The data were analysed using the SPSS 17.0 software package (SPSS, Chicago, IL, USA). Differential expression levels of lncRNAs were compared by independent-samples $t$-test between two groups, and Fisher's exact test was used in GO and pathway analysis. All values are expressed as the mean \pm standard deviation from three independent experiments, and a $P$ value of $<0.05$ was considered statistically significant.

\section{Authors' contributions}

Ling-Mei Qian and Chun Zhu designed and supervised experiments; Yu-Mei Chen and $\mathrm{Hua} \mathrm{Li}$ performed all the experiments; Yi Fan prepared animal and tissue and extracted RNA; Qi-Jun Zhang, Xing $\mathrm{Li}, \mathrm{Li}-\mathrm{Jie} \mathrm{Wu}$ and $\mathrm{Zi}$-jie Chen contributed to the data analysis; Yu-Mei Chen organized, designed, and wrote the paper; Yu-Mei Chen and Chun Zhu critically revised the manuscript. All authors reviewed the final manuscript.

\section{ACKNOWLEDGMENTS}

This study was supported by grants from the National Natural Science Foundation of China (No. 81370278 and No. 81570209) and Nanjing Medical Science and Technique Development Foundation (Ykk15148).

\section{CONFLICTS OF INTEREST} interests

\section{REFERENCES}

1. Bergmann O, Bhardwaj RD, Bernard S, Zdunek S, BarnabéHeider F, Walsh S, Zupicich J, Alkass K, Buchholz BA, Druid H, Jovinge S, Frisén J. Evidence for cardiomyocyte renewal in humans. Science. 2009; 324:98-102.

2. Beltrami AP, Urbanek K, Kajstura J, Yan SM, Finato N, Bussani R, Nadal-Ginard B, Silvestri F, Leri A, Beltrami CA, Anversa P. Evidence that human cardiac myocytes divide after myocardial infarction. N Engl J Med. 2001; 344:1750-1757.

3. Senyo SE, Steinhauser ML, Pizzimenti CL, Yang VK, Cai L, Wang M, Wu TD, Guerquin-Kern JL, Lechene CP, Lee RT. Mammalian heart renewal by pre-existing cardiomyocytes. Nature. 2013; 493:433-436.

4. Porrello ER, Mahmoud AI, Simpson E, Hill JA, Richardson JA, Olson EN, Sadek HA. Transient regenerative potential of the neonatal mouse heart. Science.2011; 331:1078-1080.

5. Porrello ER, Mahmoud AI, Simpson E, Johnson BA, Grinsfelder D, Canseco D, Mammen PP, Rothermel BA, Olson EN, Sadek HA. Regulation of neonatal and adult mammalian heart regeneration by the miR-15 family. Proc Natl Acad Sci USA 2013; 110:187-192.

6. Strungs EG, Ongstad EL, O'Quinn MP, Palatinus JA, Jourdan LJ, Gourdie RG. Cryoinjury models of the adult and neonatal mouse heart for studies of scarring and regeneration. Methods Mol Biol 2013; 1037:343-353.

7. Liu M, Zhu JG, Yu ZB, Song GX, Shen YH, Liu YQ, Zhu C, Qian LM. Identification of differentially expressed genes involved in transient regeneration of the neonatal C57BL/6J mouse heart by digital gene expression profiling. Mol Med Rep. 2014; 9:2111-2116.

8. Batista PJ, Chang HY. Long noncoding RNAs: cellular address codes in development and disease. Cell 2013; 152:1298-1307.

9. Schmitz SU, Grote P, Herrmann BG. Mechanisms of long noncoding RNA function in development and disease. Cell Mol Life Sci. 2016; 73:2491-2509.

10. Grote P, Wittler L, Hendrix D, Koch F, Währisch S, Beisaw A, Macura K, Bläss G, Kellis M, Werber M, Herrmann BG. The tissue-specific lncRNA Fendrr is an essential regulator of heart and body wall development in the mouse. Dev Cell. 2013; 24:206-214.

11. Ishii N, Ozaki K, Sato H, Mizuno H, Saito S, Takahashi A, Miyamoto Y, Ikegawa S, Kamatani N, Hori M, Saito S, Nakamura Y, Tanaka T. Identification of a novel non-coding RNA, MIAT, that confers risk of myocardial infarction. J Hum Genet. 2006; 51:1087-1099.

12. Wang K, Liu F, Zhou LY, Long B, Yuan SM, Wang Y, Liu CY, Sun T, Zhang XJ, Li PF. The long noncoding RNA CHRF regulates cardiac hypertrophy by targeting miR-489. Circ Res. 2014; 114:1377-1388. 
13. Loewer S, Cabili MN, Guttman M, Loh YH, Thomas K, Park IH, Garber M, Curran M, Onder T, Agarwal S, Manos PD, Datta S, Lander ES, et al. Large intergenic non-coding RNA-RoR modulates reprogramming of human induced pluripotent stem cells. Nat Genet. 2010; 42:1113-1117.

14. Yin Y, Wang H, Liu K, Wang F, Ye X, Liu M, Xiang R, Liu N, Liu L. Knockdown of H19 enhances differentiation capacity to epidermis of parthenogenetic embryonic stem cells. Curr Mol Med. 2014; 14:737-748.

15. Trapnell C, Pachter L, Salzberg SL. TopHat: discovering splice junctions with RNA-Seq. Bioinformatics. 2009; 25:1105-1111.

16. Trapnell C, Williams BA, Pertea G, Mortazavi A, Kwan G, van Baren MJ, Salzberg SL, Wold BJ, Pachter L. Transcript assembly and quantification by RNA-Seq reveals unannotated transcripts and isoform switching during cell differentiation. Nat Biotechnol. 2010; 28:511-515.

17. Balakrishnan R, Harris MA, Huntley R, Van Auken K, Cherry JM. A guide to best practices for Gene Ontology (GO) manual annotation. Database (Oxford). 2013; 2013:bat054.

18. Yu G, Yao W, Wang J, Ma X, Xiao W, Li H, Xia D, Yang Y, Deng K, Xiao H, Wang B, Guo X, Guan W, et al. LncRNAs expression signatures of renal clear cell carcinoma revealed by microarray. PLoS One. 2012; 7:e42377.

19. Amaral PP, Mattick JS. Noncoding RNA in development. Mamm Genome 2008; 19:454-492.

20. Martianov I, Ramadass A, Serra Barros A, Chow N, Akoulitchev A. Repression of the human dihydrofolate reductase gene by a non-coding interfering transcript. Nature. 2007; 445:666-670.

21. Feng J, Bi C, Clark BS, Mady R, Shah P, Kohtz JD. The Evf-2 noncoding RNA is transcribed from the Dlx$5 / 6$ ultraconserved region and functions as a Dlx-2 transcriptional coactivator. Genes Dev. 2006; 20:1470-1484.

22. Liao Q, Liu C, Yuan X, Kang S, Miao R, Xiao H, Zhao G, Luo H, Bu D, Zhao H, Skogerbø G, Wu Z, Zhao Y. Largescale prediction of long non-coding RNA functions in a coding-non-coding gene co-expression network. Nucleic Acids Res. 2011; 39:3864-3878.

23. Guo X, Gao L, Liao Q, Xiao H, Ma X, Yang X, Luo H, Zhao G, Bu D, Jiao F, Shao Q, Chen R, Zhao Y. Long non-coding RNAs function annotation: a global prediction method based on bi-colored networks. Nucleic Acids Res. 2013; 41:e35.

24. Beauchemin M, Smith A, Yin VP. Dynamic microRNA101a and Fosab expression controls zebrafish heart regeneration. Development. 2015; 142:4026-4037.

25. Ebelt H, Zhang Y, Kampke A, Xu J, Schlitt A, Buerke M, Müller-Werdan U, Werdan K, Braun T. E2F2 expression induces proliferation of terminally differentiated cardiomyocytes in vivo. Cardiovasc Res 2008; 80:219-226.

26. Yoshizumi M, Lee WS, Hsieh CM, Tsai JC, Li J, Perrella MA, Patterson C, Endege WO, Schlegel R,
Lee ME. Disappearance of cyclin A correlates with permanent withdrawal of cardiomyocytes from the cell cycle in human and rat hearts. J Clin Invest 1995; 95:2275-2280.

27. Bersell K, Arab S, Haring B, Kühn B. Neuregulin1/ErbB4 signaling induces cardiomyocyte proliferation and repair of heart injury. Cell. 2009; 138:257-270.

28. Di Stefano V, Giacca M, Capogrossi MC, Crescenzi M, Martelli F. Knockdown of cyclin-dependent kinase inhibitors induces cardiomyocyte re-entry in the cell cycle. J Biol Chem. 2011; 286:8644-8654.

29. Koenig SN, Bosse K, Majumdar U, Bonachea EM, Radtke F, Garg V. Endothelial Notch1 Is Required for Proper Development of the Semilunar Valves and Cardiac Outflow Tract. J Am Heart Assoc. 2016; 22:5. pii: e003075.

30. Singh R, Hoogaars WM, Barnett P, Grieskamp T, Rana MS, Buermans H, Farin HF, Petry M, Heallen T, Martin JF, Moorman AF, 't Hoen PA, Kispert A, et al. Tbx2 and Tbx3 induce atrioventricular myocardial development and endocardial cushion formation. Cell Mol Life Sci. 2012; 69:1377-89.

31. Breckwoldt K, Weinberger F, Eschenhagen T. Heart regeneration. Biochim Biophys Acta. 2015; 1863:1749-1759.

32. Klattenhoff CA, Scheuermann JC, Surface LE, Bradley RK, Fields PA, Steinhauser ML, Ding H, Butty VL, Torrey L, Haas S, Abo R, Tabebordbar M, Lee RT, et al. Braveheart, a long noncoding RNA required for cardiovascular lineage commitment. Cell 2013; 152:570-583.

33. Werber M, Wittler L, Timmermann B, Grote $P$, Herrmann BG. The tissue-specific transcriptomic landscape of the mid-gestational mouse embryo. Development. 2014; 141:2325-2330.

34. Ounzain S, Micheletti R, Arnan C, Plaisance I, Cecchi D, Schroen B, Reverter F, Alexanian M, Gonzales C, Ng SY, Bussotti G, Pezzuto I, Notredame C, et al. CARMEN, a human super enhancer-associated long noncoding RNA controlling cardiac specification, differentiation and homeostasis. J Mol Cell Cardiol. 2015; 89:98-112.

35. Ounzain S, Pedrazzini T. The promise of enhancerassociated long noncoding RNAs in cardiac regeneration. Trends Cardiovasc Med. 2015; 25:592-602.

36. Folmes CD, Terzic A. Metabolic determinants of embryonic development and stem cell fate. Reprod Fertil Dev. 2014; 27:82-88.

37. Jopling C, Suñe G, Morera C, Izpisua Belmonte JC. p38alpha MAPK regulates myocardial regeneration in zebrafish. Cell Cycle. 2012; 11:1195-1201.

38. Xin M, Kim Y, Sutherland LB, Murakami M, Qi X, McAnally J, Porrello ER, Mahmoud AI, Tan W, Shelton JM, Richardson JA, Sadek HA, Bassel-Duby R, et al. Hippo pathway effector Yap promotes cardiac regeneration. Proc Natl Acad Sci USA 2013; 110: 13839-13844. 
39. Heallen T, Zhang M, Wang J, Bonilla-Claudio M, Klysik E, Johnson RL, Martin JF. Hippo pathway inhibits Wnt signaling to restrain cardiomyocyte proliferation and heart size. Science. 2011; 332:458-461.

40. Heallen T, Morikawa Y, Leach J, Tao G, Willerson JT, Johnson RL, Martin JF: Hippo signaling impedes adult heart regeneration. Development. 2013; 140:4683-4690.

41. White IA, Gordon J, Balkan W, Hare JM: Sympathetic Reinnervation Is Required for Mammalian Cardiac Regeneration. Circ Res. 2015; 117:990-994.

42. Jiang YH, Jiang P, Yang JL, Ma DF, Lin HQ, Su WG, Wang Z, Li X. Cardiac Dysregulation and Myocardial Injury in a 6-Hydroxydopamine-Induced Rat Model of Sympathetic Denervation. PLoS Onel. 2015; 10:e0133971.

43. Oldham MC, Konopka G, Iwamoto K, Langfelder P, Kato T, Horvath S, Geschwind DH. Functional organization of the transcriptome in human brain. Nat Neurosci. 2008; 11:1271-1282.
44. Volpe M, Shpungin S, Barbi C, Abrham G, Malovani H, Wides R, Nir U. trnp: A conserved mammalian gene encoding a nuclear protein that accelerates cell-cycle progression. DNA Cell Biol. 2006; 25:331-339.

45. Liu D, He M, Yi B, Guo WH, Que AL, Zhang JX: Pim3 protects against cardiomyocyte apoptosis in anoxia/ reoxygenation injury via p38-mediated signal pathway. Int J Biochem Cell Biol. 2009; 41:2315-2322.

46. Oikonomopoulos A, Sereti KI, Conyers F, Bauer M, Liao A, Guan J, Crapps D, Han JK, Dong H, Bayomy AF, Fine GC, Westerman K, Biechele TL, et al. Wnt signaling exerts an antiproliferative effect on adult cardiac progenitor cells through IGFBP3. Circ Res. 2011; 109:1363-74.

47. Ross RS, Borg TK. Integrins and the myocardium. Circ Res. 2001; 88:1112-1119. 